\title{
Some Simple Mathematical Models in Epilepsy
}

\author{
E Ahmed* \\ Mathematics Department, Faculty of Science, Mansoura, Egypt \\ *Corresponding author: E Ahmed, Mathematics Department, Faculty of Science, Mansoura, Egypt
}

\section{Abstract \\ Epilepsy is a chronic disorder of the brain that affects people of all ages. Here epileptic seizures equations are related to the telegraph equation.}

\section{Introduction}

\section{Some Basic Facts about Epilepsy}

Epilepsy is a chronic disorder of the brain that affects people of all ages. Approximately 50 million people worldwide have epilepsy, making it one of the most common neurological diseases globally. People with epilepsy respond to treatment approximately $70 \%$ of the time. About three fourths of people with epilepsy living in lowand middle- income countries do not get the treatment they need. Therefore, using different approaches to study epilepsy is useful [1].

\subsection{Telegraph Equation in Epilepsy}

Telegraph equation [2,3] is a generalization of diffusion equation where the standard diffusion equation depends on the continuity equation and Fick's law

$$
j(x, t)=-D \partial c(x, t) / \partial x
$$

Where,

$\mathrm{j}$ is the diffusing object (e. g. technology, concept, etc...),

$\mathrm{c}$ is the distribution function of this object and

D is the diffusion constant.

The resulting standard diffusion equation is

$$
\partial c(x, t) / d t=D \partial 2 c(x, t) / \partial x 2
$$

where is the second order differentiation w. r. t. position $\mathrm{x}$.

A basic weakness of this equation is that the flux $\mathrm{j}$ reacts simultaneously to the gradient of $\mathrm{c}$ and consequently an unbounded propagation speed is assumed. To solve this problem Fick's law is replaced by

$$
j+T \partial j / \partial t=D \partial c / \partial x
$$

where $\mathrm{T}$ is a time constant which measures the memory or delay effect. Thus, one gets the telegraph equation:

$T \partial 2 c / \partial t 2+(1-T d f / d c) \partial c / \partial t=D \partial 2 c / \partial x 2+f(c)$

Applying this equation to the case

$$
f(c)=\alpha c+\beta c^{\wedge} 2+\varepsilon c^{\wedge} 3
$$

and making the identifications

$$
\begin{aligned}
& c(t, x)=\sin (\lambda x) g(t), T=1 /\left(\gamma^{\wedge} 2\right), \\
& 2 / \gamma+c 2 t_{0}=1-T \alpha, \alpha-\gamma^{\wedge} 2=c_{1}+c_{2}
\end{aligned}
$$

one regains equations 1-4 in [4] where t0 is small and we made the expansion

$$
g\left(t-t_{0}\right) \sim g(t)-t_{0} d g / d t
$$

These equations are used to explain dynamical properties of epileptic seizures.

\section{References}

1. WHO (2018) Epilepsy. Geneva, Switzerland.

2. R Metzler, Albert Compte (1997) The generalized Cattaneo equation for the description of anomalous transport processes. J Phys A 30(21): 7277.

3. E Ahmed, S Z Hassan (2000) ZNF 55a: 669.

4. JW Kim, JA Roberts, PA Robinson (2009) Dynamics of epileptic seizures: Evolution, spreading, and suppression. J. Theoretical Biology 257(4): 527-532. 
(C) This work is licensed under Creative To Submit Your Article Click Here: Submit Article

DOI: 10.32474/CTBB.2018.01.000109

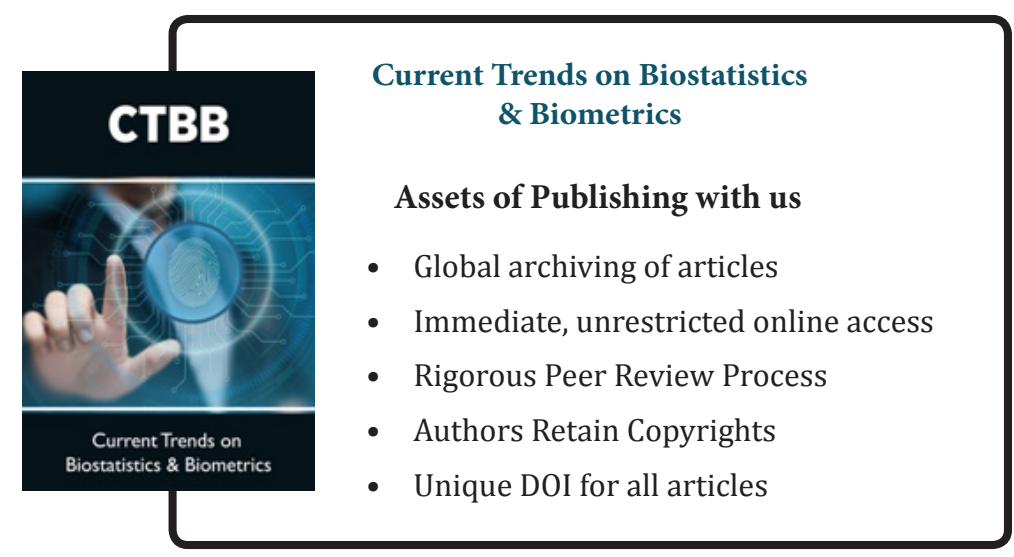

